Journal of Applied Fluid Mechanics, Vol. 15, No. 1, pp. 85-98, 2022.

Available online at www.jafmonline.net, ISSN 1735-3572, EISSN 1735-3645.

https://doi.org/10.47176/jafm.15.0.32762

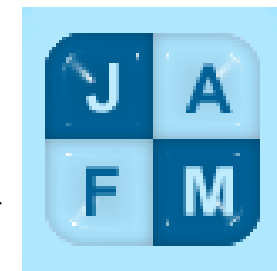

\title{
A Comparative Study of the Buoyancy-Opposed Wall Jet using Different Turbulent Models
}

\author{
X. $\mathrm{Nie}^{1 \dagger}$, Z. H. Zhu ${ }^{1}$, H. B. Liao ${ }^{1}$, Y. Z. Zhang ${ }^{1}$ and J. R. Xu ${ }^{2}$ \\ ${ }^{1}$ School of Mechanical Engineering, Hangzhou Dianzi University, Hangzhou, Zhejiang, 310018, China \\ ${ }^{2}$ School of Science, Hangzhou Dianzi University, Hangzhou, Zhejiang, 310018, China \\ †Corresponding Author Email: nx2000@hdu.edu.cn
}

(Received March 8, 2021; accepted July 19, 2021)

\begin{abstract}
A comparative study of the buoyancy-opposed wall jet has been carried out using RANS methods (including RNG model, Realizable $k-\varepsilon$ model, and two low Reynolds number $k-\varepsilon$ models) and LES methods (including the subgrid scale model developed by Smagorinsky et al.(1963), Germano et al. (1991) and Kim et al. (1997)). The capability of each turbulence model to predict the flow field and temperature field in mixing stage was investigated. The results show that the $k$ - $\varepsilon$ series model can accurately predict the velocity distribution of flow field under isothermal case. However, in the case of buoyancy, due to the assumption of turbulent normal stress isotropy, the trend of temperature change in the mixing region and transition position existed an obvious deviation with experimental data. The LES methods, solved directly the large scale vortices, take into account the influence of turbulence stress anisotropy in the mixing region on the temperature change and capture the temperature change trend over the whole domain accurately. Due to the application of the subgrid kinetic energy transport equation, KET model has certain advantages in numerical simulation of similar engineering flow phenomenon.
\end{abstract}

Keywords: Buoyancy effect; Wall jet; LES; Sub-grid kinetic energy transport equation

\begin{tabular}{|c|c|c|c|}
\hline \multicolumn{4}{|c|}{ NOMENCLATURE } \\
\hline$C_{p}$ & specific heat & $t$ & fluctuation temperature \\
\hline$C_{\varepsilon 1}, C_{\varepsilon 2}, C_{\mu}$ & turbulence model constants & $U$ & time-averaged velocity \\
\hline$D, E$ & additional source items & $\overline{u_{i}}$ & fluctuation velocity \\
\hline$f_{\mu}, f_{1}, f_{2}$ & damping functions & $w$ & nozzle width \\
\hline$h$ & surface heat transfer coefficient & $x, y$ & coordinates \\
\hline$I$ & turbulence intensity & $Y_{\text {plus }}$ & dimensionless distance \\
\hline$k$ & turbulent kinetic energy & $\alpha$ & thermal conductivity \\
\hline$P$ & static pressure & $\alpha_{t}$ & turbulent thermal diffusivity \\
\hline$P_{k}$ & $\begin{array}{l}\text { turbulent kinetic energy production } \\
\text { term }\end{array}$ & $\delta$ & $\begin{array}{l}\text { distance normal to the wall in the } \\
\text { outer layer }\end{array}$ \\
\hline$R e$ & $\begin{array}{l}\text { Jet Reynolds number based on jet inlet } \\
\text { velocity and hydraulic diameter }\end{array}$ & $\varepsilon$ & turbulent dissipation rate \\
\hline$R e_{T}, R_{y}$ & turbulence Reynolds numbers & $\varepsilon$ & $\begin{array}{l}\text { modified isotropic dissipation rate } \\
\text { related to } \varepsilon\end{array}$ \\
\hline$T$ & temperature & $\mu, \mu_{t}$ & laminar and eddy viscosities \\
\hline$T_{i n f}$ & Jet temperature & $v, v_{t}$ & $\begin{array}{l}\text { laminar and eddy kinematic } \\
\text { viscosities }\end{array}$ \\
\hline$T_{t}$ & turbulence time scale & $\rho$ & density \\
\hline$T_{\text {wall }}$ & wall temperature & $\sigma_{k}, \sigma_{\varepsilon}, \sigma_{t}$ & turbulent Prandtl numbers \\
\hline
\end{tabular}




\section{INTRODUCTION}

When the hotter plane jet down a vertical wall encountered the counterflowing stream, it would lead to buoyant as well as dynamic influences on the stagnation point position and the wall jet turn to flow upstream eventually. It is encountered in many industrial applications and also in nature, such as atmospheric convection and ocean current phenomenon, window defrosting and demisting (Goldman and Jaluria 1986, Kapoor and Jaluria 1989, 1991). Especially in gas-cooled nuclear reactors (He et al. 2002), the study of buoyancy opposed jet is a key point and challenge. In the past 30 years, many scholars have carried out experimental studies on the flow field and heat transfer of the buoyancy opposed jet. (Goldman et al. (1986) have carried out an experimental study of a two-dimensional buoyancy opposed wall jet and pointed out that the buoyancy effect increases with the increase of Richards number $\mathrm{Ri}\left(\mathrm{Gr} / \mathrm{Re}^{2}\right)$. Subsequently, on the basis of Goldman et al. a further research was carried out by Kapoor et al. (1989, 1991). They found the jet penetration depth and heat transfer effect decrease with the increase of buoyancy effect (Kapoor et al. 1989, 1991).

He et al. (2002) summarized the previous work, combined with the background of engineering application (gas-cooled nuclear reactor), carried out a detailed experimental study on the flow field and temperature field of the wall jet in buoyant case by using particle image velocimetry (PIV), Doppler anemometer and thermocouple. They measured the flow field and temperature change of Ri parameter in the range of $0 \sim 0.052$. In the decades that followed, their experimental data was used for verification in CFD codes which were used in industry to study such flows.

Due to the interaction of the wall jet flow and the counter-current stream, high levels of turbulent shear stress are generated in the mixing region and it further leads to a strong anisotropy. Hence, for numerical simulation research, it puts forward higher requirements for turbulent models and many scholars have carried out numerical simulation research on the buoyancy-opposed wall jet (Addad et al. 2004, Craf et al. 2004, Rathore and Das 2016). Craft et al. (2004) have applied the low-Reynolds number model of Launder et al. (1974), the standard $k-\varepsilon$ model and the two second-moment closures with standard wall function and analytical wall function (AWF) (Craft et al. 2002) to carry out the numerical simulation of buoyancy-opposed wall jet. They obtained the satisfactory calculation in iosthermal case by using AWF (Craft et al. 2002) and in the meanwhile they pointed out that the buoyant flow in the buoyant cases was a modelling challenges for turbulent models. The standard $k-\varepsilon$ model is widely used in computational fluid dynamics and performs quite well for boundary layer flows. But there exist a high mean shear rate or a massive separation in the mixing region, the standard $k-\varepsilon$ model may overestimate the eddy viscosity due to the standard eddy viscosity formulation. To fix this problem, the RNG $k-\varepsilon$ model and Realizable $k-\varepsilon$ model (Speziale and Thangam 1992, Shih et al. 1995) is put forward by modifying the dissipation rate equation on the basis of the the standard $k-\varepsilon$ model.

Huai et al. (2010) have applied the RNG $k-\varepsilon$ model and Realizable $k-\varepsilon$ model to carry out the numerical simulation of multiple tandem jets and buoyant wall jet (Wen-xin et al. 2010, Meng et al. 2018) and proved their effectiveness. In particular, they obtained the satisfactory calculation in both multiple tandem jets and buoyant wall jet by using Realizable $k-\varepsilon$ model.

Compared with the $k-\varepsilon$ models that uses the wall function to bridge the viscous and buffer layers, the low-Reynolds number $k-\varepsilon$ model has theoretical advantages to the numerical simulation of the flow field and the heat transfer in the boundary layer (Xin et al. 2021). Rathore and Das (2016) successfully simulated the buoyancy-opposed wall jet by using the low Reynolds number $k-\varepsilon$ model proposed by Yang and Shih (1993). They further studied and analyzed the calculation results under buoyancy condition by combining with turbulent kinetic energy and turbulent dissipation rate.

Since Large eddy simulation (LES) contains timedependent information such as evolution of large eddies, instantaneous fluctuations, time traces of quantities, spectra and two-point correlations, it can offer more flow and turbulence information than traditional methods of turbulence prediction. Addad et al. (2004) have numerically studied the buoyancyopposed wall jet using the LES, they presented the fairly satisfactory results for the isothermal and moderately buoyant cases. However, they adopted the relatively sparse grid model due to the limitation of computer ability, and the experimental data utilized for velocity comparisons are absent in $\mathrm{He}$ et al. (2002) Those factors lead to the computational results lack credibility.

Li et al. (2011) studied the interaction between a wall jet and an offset jet using the LES models including the models developed by Germano and Piomelli, and Kim and Menon. They pointed out the LES model developed by Kim and Menon has a clear advantages in predicting the mean stream-wise velocity, the halfwidth of the velocity and the decay of the maximum velocity and further study the turbulence mechanism by coherent structure and the correlation function, probability density function of the fluctuating velocity. After that, (Li et al. 2012) successfully simulated the vertical buoyant jet in an open channel with emergent vegetation by using the LES model developed by Guo et al. (2006). In their study, the temporal and spatial evolution of vortex structures was analysed in detail to elucidate the effect of vegetation on jet development. Regretfully, they 
mentioned less to the influence of buoyancy to turbulence structures.

In recent years, with the development of computer ability and turbulence model theory, it offers more resource and approaches to carry out the numerical simulation of turbulent research. Moreover, the above numerical research results all get a satisfactory calculation in iosthermal case, but failed to simulation buoyancy case with strong buoyant effects and paid little attention the influence to temperature field. In the meanwhile, literature survey shows that, thereafter no archival published literature exists continuing a further exploration of buoyancy-opposed wall jet. In order to bridge this gap, Reynolds Averaged Navier-Stokes (RANS) models and Large eddy simulation (LES) are used to carry out the three-dimensions numerical simulations of the buoyancy-opposed wall jet with a fine grid. Subsequently, the present study showcases systematically the numerical results compared with the experimental results of He et al. (2002) and investigate the capabilities of turbulence model for predicting flow field and temperature field influenced by buoyancy effect.

The RANS models were those developed by Speziale and Thangam(thereafter referred to as RNG $k-\varepsilon$ ) (Speziale and ZhiWei 1992); Shih and Liou (thereafter referred to as Realizable $k-\varepsilon$ ) (Shih et al. 1995); Launder and Sharma(thereafter referred to as LS) (Launder et al. 1974); Yang and Shih (thereafter referred to as YS) (Yang and Shih 1993).

The LES models were those developed by Smagorinsky and Lilly (thereafter referred to as SM) (Smagorinsky 1963); Germano and Piomelli (thereafter referred to as DSM) (Germano et al. 1991); Kim and Menon(thereafter referred to as KET) (Kim et al. 1997).

\section{MATHEMATICAL MODEL}

\subsection{RANS Models}

In the present study, it is assumed that the fluid (air) is essentially incompressible and Newtonian with temperature-dependent fluid properties. The wall jet flow is also assumed to be in a time-averaged quasisteady state.

Therefore, the continuity equation is

$\frac{\partial U_{i}}{\partial X_{i}}=0$

and the Reynolds averaged Navier-Stokes equation is

$U_{i} \frac{\partial U_{j}}{\partial x_{i}}=-\frac{1}{\rho} \frac{\partial P}{\partial x_{i}}+\frac{\partial}{\partial x_{i}}\left(v \frac{\partial U_{i}}{\partial x_{j}}-\overline{u_{i} u_{j}}\right)+B_{T i}$

The time-averaged energy equation is
$U_{i} \frac{\partial T}{\partial x_{i}}=\frac{\partial}{\partial x_{i}}\left(\frac{\lambda}{c_{p} \rho} \frac{\partial T}{\partial x_{j}}-\frac{\overline{u_{i} t}}{c_{p}}\right)$

In the above equation, the Reynolds stress terms are assumed to be proportional to the local mean velocity gradients through Boussinesq's eddy transport approximation, and the fluctuation term of temperature equation are closed in same way.

$\overline{u_{i} u_{j}}=2 / 3 k \delta_{i j}-v_{t}\left(\frac{\partial U_{i}}{\partial x_{j}}+\frac{\partial U_{j}}{\partial x_{i}}\right)$

$\overline{u_{i}} t=-\alpha_{t} \frac{\partial \bar{T}}{\partial x_{j}}$

where the turbulent eddy viscosity and turbulent thermal diffusivity are given by

$$
\begin{aligned}
& v_{t}=\frac{C_{\mu} f_{\mu} k^{2}}{\varepsilon} \\
& \alpha_{t}=\frac{v_{t}}{\sigma_{t}}
\end{aligned}
$$

The transport equations for the k- $\varepsilon$ models are

$$
\begin{aligned}
& U_{i} \frac{\partial k}{\partial x_{i}}=\frac{\partial}{\partial x_{j}}\left[\left(v+\frac{v_{t}}{\sigma_{k}}\right) \frac{\partial k}{\partial x_{j}}\right]+P_{k}-\tilde{\varepsilon}-D+G_{k} \\
& U_{i} \frac{\partial \tilde{\varepsilon}}{\partial x_{i}}=\frac{\partial}{\partial x_{j}}\left[\left(v+\frac{v_{t}}{\sigma_{\varepsilon}}\right) \frac{\partial \tilde{\varepsilon}}{\partial x_{j}}\right]+ \\
& C_{\varepsilon 1} f_{1} \frac{1}{T_{t}}\left(P_{k}+G_{k}\right)-C_{\varepsilon 2} f_{2} \frac{\tilde{\varepsilon}}{T_{t}}+E
\end{aligned}
$$

Where $U$ is velocity; $\rho$ is the density; $P$ is the pressure; $\lambda$ is the thermal conductivity; $c_{p}$ is the specific heat capacity; $\overline{u_{i}}$ is fluctuation velocity, $\bar{t}$ is fluctuation temperature, the model parameters $f_{\mu}$, $f_{1}, f_{2}, \tilde{\varepsilon}, D$ and $E$ vary with the different models; $f_{\mu}$, $f_{1}$ and $f_{2}$ are the damping functions of the local turbulent Reynolds number, $\tilde{\varepsilon}$ is the modified isotropic dissipation rate related to $\varepsilon, D$ and $E$ are additional source items, and $P_{k}$ is turbulent kinetic energy production term. In the low-Reynolds number $k-\varepsilon$ models, the turbulent eddy viscosity coefficient is defined by Eq. (6) where $C_{\mu}$ is a constant and $f_{\mu}$ is a damping function. The turbulent thermal diffusivity coefficient $\alpha_{t}$ is obtained by Eq. (7) by turbulent Prandtl number $\sigma_{t}$. The Prandtl number and turbulent Prandtl number is taken as 0.71 and 0.9 for air and water (Kays et al. 1994), respectly. The $T_{t}$ in Eq. (9) is the turbulence time scale expressed as $k / \varepsilon$. The $B_{T i}$ in Eq. (2) is the buoyancy term expressed as $B_{T i}=g_{i}\left(\rho-\rho_{\text {ref }}\right)$. The $G_{k}$ in $k-\varepsilon$ transport Eq. (5) and Eq. (6) also represent buoyancy term expressed as $G_{k}=\overline{\rho^{\prime} u_{i} g_{i}}=$ $-\rho \beta g_{i} \overline{u_{i} t}$ and the $\beta$ is the volume expansion coefficient of fluid.

The RNG $k-\varepsilon$ and Realizable $k-\varepsilon$ model relate the $C_{\varepsilon l}$ coefficient to the time average strain rate $S_{i j}$ by modifying dissipation rate equation and the relevant 
model constants $C_{\varepsilon 1}, C_{\varepsilon 2}, C_{\mu}, \sigma_{k}$ and $\sigma_{\varepsilon}$ in RNG $k-\varepsilon$ and Realizable $k-\varepsilon$ are also modified (Speziale et al. 1992, Shih et al. 1995), which are different from the standard $k-\varepsilon$ model. See Table 1 for details. The damping functions $f_{\mu}, f_{1}$ and $f_{2}$ are vary with different Low-Reynolds Number $k$ - $\varepsilon$ models, See Table 2 for details. It is noted that the friction velocity $u_{\tau}$ is not included in the damping function definition of the above two low Reynolds number models, so those models can be used for the flow with boundary transition separation (Xin et al. 2021).

\subsection{LES Models}

The non-uniform box filter function was used to filter the N-S equation.

$$
\begin{aligned}
& \varphi_{i}=\bar{\varphi}_{i}+\varphi_{i}^{\prime} \\
& \mathrm{G}\left(x-x^{\prime}\right)= \begin{cases}1 / \Delta, & |x-\xi| \leq \Delta / 2 \\
0, & |x-\xi|>\Delta / 2\end{cases}
\end{aligned}
$$

Table 1. Summary of model constants and source term in governing equations

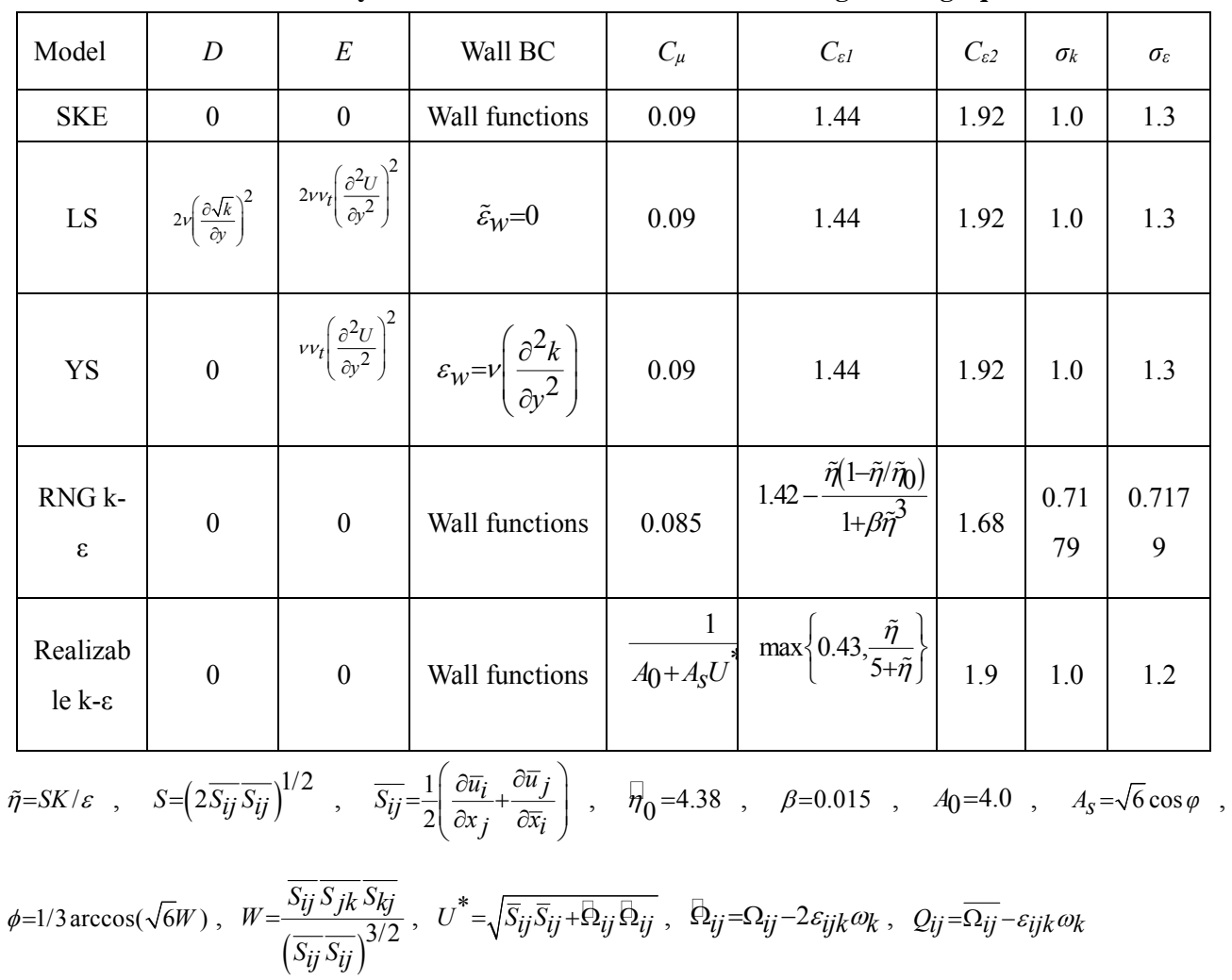

\begin{tabular}{|c|c|c|c|}
\hline Model & $f_{\mu}$ & $f_{l}$ & $f_{2}$ \\
\hline SKE & 1.0 & 1.0 & 1.0 \\
\hline LS & $\exp \left[-3.4 /\left(1+\operatorname{Re}_{t} / 50\right)^{2}\right]$ & 1.0 & $1-0.3 \exp \left(-\operatorname{Re}_{t}^{2}\right)$ \\
\hline YS & $\begin{array}{l}\left(1+1 / \operatorname{Re}_{t}^{0.5}\right)[1-\exp (-1.5 \\
\times 10^{-4}-\operatorname{Re}_{y}-5 \times 10^{-7} \operatorname{Re}_{y}^{3} \\
\left.\left.-10^{-10} \operatorname{Re}_{y}^{5}\right)\right]^{0.5}\left(1+\operatorname{Re}_{t}^{0.5}\right)\end{array}$ & $\operatorname{Re}_{t}^{0.5} /\left(1+\operatorname{Re}_{t}^{0.5}\right)$ & $\operatorname{Re}_{t}^{0.5} /\left(1+\operatorname{Re}_{t}^{0.5}\right)$ \\
\hline RNG k- $\varepsilon$ & 1.0 & 1.0 & 1.0 \\
\hline Realizable k- $\varepsilon$ & 1.0 & 1.0 & 1.0 \\
\hline
\end{tabular}

Table 2. Summary of damping functions appearing in the governing equations

$\operatorname{Re}_{t}=\frac{k^{2}}{v \varepsilon}, \operatorname{Re}_{y}=\frac{y k^{1 / 2}}{v}, y^{*}=\frac{y u_{\varepsilon}}{v}, u_{\varepsilon}=(v \varepsilon)^{0.25}, \tilde{\varepsilon}=\varepsilon+D$ 
X. Nie et al. / JAFM, Vol. 15, No. 1, pp. 85-98, 2022.

$\bar{\varphi}(x, t)=\int_{V} \varphi\left(\xi_{x}, t\right) G\left(x-\xi_{x}\right) d \xi_{x}$

Continuous equation after filtering:

$\frac{\partial \bar{u}_{i}}{\partial x_{i}}=0$

Momentum equation:

$\frac{\partial \bar{u}_{i}}{\partial t}+\frac{\partial \bar{u}_{i} \bar{u}_{j}}{\partial x_{j}}=-\frac{1}{\rho} \frac{\partial P}{\partial x_{i}}+v \frac{\partial^{2} \bar{u}_{i}}{\partial x_{j} \partial x_{j}}-\frac{\partial \tau_{i j}}{\partial x_{j}}+B_{T i}$

where:

$\tau_{i j}=\overline{u_{i}} \overline{u_{j}}-\overline{u_{i} u_{j}}=-2 v_{t} \overline{S_{i j}}+\frac{1}{3} \tau_{k k} \delta_{i j}$

$\frac{\partial \bar{T}}{\partial x_{i}}+\frac{\partial\left(\overline{T u_{j}}\right)}{\partial x_{j}}=\frac{1}{\operatorname{Re}} \frac{1}{\operatorname{Pr}} \frac{\partial^{2} \bar{T}}{\partial x_{j}^{2}}-\frac{\partial Q_{j}}{\partial x_{j}}$

Where "-" represents filtered variables; Re is the Reynolds number expressed as $R e=u d / v$; $\operatorname{Pr}$ is the Prandtl number expressed as $\operatorname{Pr}=v / \alpha$; Where $d$ is characteristic length; $v$ is the Kinematic viscosity coefficient; $\alpha$ is the Thermal diffusivity; $\boldsymbol{\tau}_{i j}$ represents the subgrid turbulent stress, which need to construct subgrid model to solve. The subgrid models developed by Smagorinsky et al. and Germano et al . can be found in literature (Smagorinsky 1963, Germano et al. 1991). $Q_{j}$ represents turbulent heat flux, which can be sloved by turbulent Prandtl number $\sigma_{t}$ similar to RANS method.

The subgrid model developed by Kim and Menon establishs a turbulent energy transport equation base on subgrid scale (Kim et al. 1997). The specific expression is as follows:

$$
\begin{gathered}
\frac{\partial \bar{k}_{s g s}}{\partial t}+\frac{\partial \bar{u}_{j} \bar{k}_{s g s}}{\partial x_{j}}=-\tau_{i j} \frac{\partial \bar{u}_{i}}{\partial x_{j}} \\
-C_{\varepsilon} \frac{k_{s g s}^{1 / 2}}{\Delta f}+\frac{\partial}{\partial x_{j}}\left(\frac{v_{t}}{\sigma_{k}} \frac{\partial k_{s g s}}{\partial x_{j}}\right)
\end{gathered}
$$

where $k_{s g s}$ represents subgrid turbulent energy expressed as:

$k_{s g s}=\frac{1}{2}\left(\overline{u_{k} u_{k}}-\overline{u_{k}} \overline{u_{k}}\right)$

hence, $\boldsymbol{\tau}_{i j}$ can be expressed as:

$\tau_{i j}=\frac{2}{3} k_{s g s} \delta_{i j}-2 C_{k} k_{S g s}^{1 / 2} \Delta_{f} \bar{S}_{i j}$

where $v_{t}=C_{k} k_{s g s}^{1 / 2} \Delta_{f}, C_{k}$ and $C_{\varepsilon}$ are dynamic parameters; $\sigma_{k}$ usually take value as 1 .

\section{Problem Description}

\subsection{Simulation details and boundary conditions}

The geometrical configuration of the problem under investigation is similar to that used in the experimental work of He et al. (2002). The wall jet is injected into the channel from a narrow slot with $0.018 \mathrm{~m}$ width located on the right hand side of the channel. The counter-current stream, entering from bottom of the channel, interacts with the jet. The combined jet and countercurrent stream leaves from top of the channel. The working fluid is water. The experimental configuration of He et al. (2002) is shown in Fig.1.

For isothermal case, the lengths of the domain above and below the point of wall jet injection consistent with the experimental work are $0.6 \mathrm{~m}$ and $1.7 \mathrm{~m}$ to approach velocity profiles of experimental inlet and exit (Addad et al. 2004, Craft et al. 2004, Rathore and Das 2016).

For the buoyant case, lengths of the domain above and below the point of injection are $2.4 \mathrm{~m}$ and $1 \mathrm{~m}$, which is different with the experimental configuration of He et al. (2002) and the specific reasons are detailed in the literature (Addad et al. 2004, Craft et al. 2004, Rathore and Das 2016). The grid distribution for buoyant flow case $(\mathrm{Ri}=0.02)$ is shown in Fig. 2.

The boundary conditions were consistent with experiments, with the following specific settings: All solid walls was specified as no-slip and adiabatic; The velocity at the jet inlet is taken as $V=-0.167 \mathrm{~m} / \mathrm{s}$; The counter-flow velocity at the bottom side of the channel is $V=0.013 \mathrm{~m} / \mathrm{s}$; The temperature of wall jet and counter-flow was set according to different working conditions, see Table 3 for details. Meanwhile, turbulence intensity and length scale at nozzle exit were set to be $1 \%$ and $0.07 \mathrm{w}$ respectively. Hence, the initial value $\mathrm{k}$ at nozzel exit was calculated from $k=1.5(I U)^{2}$ and the initial value $\varepsilon$ was calculated from $\varepsilon=\left(k^{3 / 2} \cdot C_{\mu}^{3 / 4}\right) / 0.07 w$. (Jones and Chapuis 2016) In particular, for lowReynolds Number $k-\varepsilon$ models, the boundary condition for turbulent kinetic energy is $k=0$ at the solid wall. But the specific boundary condition for turbulent dissipation rate at the solid wall is vary with different low-Reynolds Number $k$ - $\varepsilon$ models, see Table 1 for details. For LES models, the Smagorinsky constant of the standard Smagorinsky model chosen is 0.1 . The initial value of turbulent physical scalar in the LES simulations was obtained from the results calculated by RANS models. And the grid spacing used in LES simulations should also satisfy the minimal ratio of local length scale $l_{m}=C_{\mu}{ }^{0.75} k^{1.5} / \varepsilon$. The grid settings applied in LES simulations will be described in detail later.

\subsection{Numerical scheme and grid independence study}

In the present work, the governing differential equations are discretized using the finite volume method on a staggered grid. The semi-implicit method for pressure-linked equations (SIMPLE) is followed to handle the velocity and pressure coupling. The second order upwind scheme were 
Table 3. Working conditions

\begin{tabular}{|c|c|c|c|c|c|c|c|}
\hline & $\mathrm{Re}$ & $\mathrm{Gr}$ & $\mathrm{Ri}$ & $V_{\mathrm{j}}(\mathrm{m} / \mathrm{s})$ & $V_{\mathrm{c}}(\mathrm{m} / \mathrm{s})$ & $T_{\mathrm{j}}\left({ }^{\circ} \mathrm{C}\right)$ & $T_{\mathrm{c}}\left({ }^{\circ} \mathrm{C}\right)$ \\
\hline 1 & 4754 & 0 & 0 & 0.167 & 0.013 & 42 & 42 \\
\hline 2 & 4754 & 23400 & 0.01 & 0.167 & 0.013 & 42 & 38 \\
\hline 3 & 4754 & 46800 & 0.02 & 0.167 & 0.013 & 42 & 34 \\
\hline
\end{tabular}

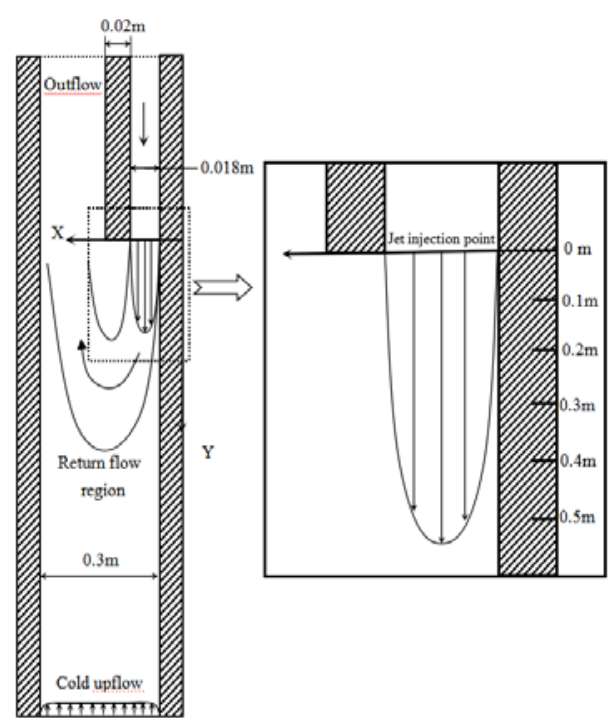

Fig. 1. Schematic diagram of jet under iosthermal condition.

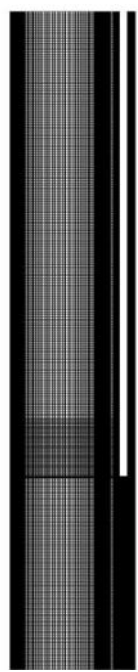

Fig. 2. Schematic diagram of grid distribution under buoyancy condition.

used to discretize the convective and diffusive terms of momentum and turbulence kinetic energy and turbulence dissipation rate. Both RANS and LES models were adopted transient calculation. For LES models, the second order implicit scheme was used to advance the time step. The every time step interval was set as $0.0001 \mathrm{~s}$ according to the grid scale and velocity scale and set 50 iterations in every time step to ensure the calculation results reach the set residual value. The convergence criteria were specified as follows: the normalized residuals of all dependent variables must be less than $10^{-6}$. After reaching the criteria, we set the residual value to $10^{-7}$ and calculate 5000 steps of iteration again. Comparing the temperature and flow field at $\mathrm{x} / \mathrm{w}=10.8$ and $\mathrm{x} / \mathrm{w}=12.7$, when maximum difference between the results is not more than $0.1 \%$, it can be considered the calculation has converged.

Due to the presence of mixing region, stagnation point, return flow resulting in a high streamline curvature, and interaction of buoyancy with the momentum of the jet, the grid density is high in those region. To resolve the near wall region with large gradients satisfactorily, finer computational grids were set near the wall, and the distance of the first grid near the wall $x_{\text {plus }}$ was taken as different value in different literature. In this paper, we set up three to five grid layers in the bottom layer $\left(x_{\text {plus }}<5\right)$ of viscous laminar flow near the wall in order to ensure the first near-wall grid points lie in the viscous sublayer for low-Reynolds number $k-\varepsilon$ models. Meanwhile, the standard $k-\varepsilon$ model, RNG $k-\varepsilon$ and Realizable $k-\varepsilon$ model all apply the enhanced wall function to solve finer grid near the wall. A proper LES must resolve all large turbulent scales in the flow, those containing most of the turbulent kinetic energy and Reynolds shear stress in each region of the flow. In this paper, the grid applyed in lowReynolds number $k-\varepsilon$ models is also suitable for large eddy simulation.

For all experiment conditions and models, the grid independence study has been carried out for the buoyant wall jet at three grid sizes viz. 11579304, 22978405 and 45367800. Figure 3 shows a comparison of the decay of downward velocity predicted by YS model at a distance of $14 \mathrm{~mm}$. It can be seen that the calculation results of three sizes of grids have a good consistency.

Considering the available computing resources and cycle of numerical simulation, the grid size of 22978405 is considered for simulation finally. See Fig. 2 for detail. 


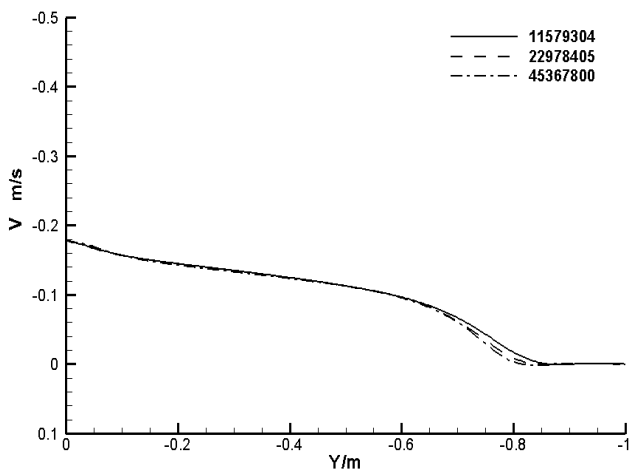

Fig. 3. Grid independence study(YS model).

\section{RESULTS AND DISCUSSION}

\subsection{RANS Models}

Figure 4 shows the comparison between the calculated results by RANS models and the experimental data of the vertical velocity components at at various heights in the test section. Where the $\mathrm{X}$-axis is the distance of the transverse flow field, and the Y-axis is the Y-direction component of the jet average velocity. The average velocity profiles of the jet is used to normalize the flow field. According to the X-direction distance in Fig.4, the flow field can be divided into three parts easily: a wall jet region, a mixing region where the wall jet flow encounter the counter-current stream and a return flow region. In the wall jet region near the right wall, the jet velocity is downwards and relatively large which decays with reduction of height. With the increase of Richardson number, this decay occurs more quickly and the depth of penetration of the downward jet is reduced. The jet velocity falls to zero in the mixing region and finally reverse flow direction in the return flow region. For the isothermal case ( $\mathrm{Ri}=0$, Fig. 4 (a)), the maximum return flow velocity appears near the left wall, and the velocity decreases slowly with the distance from the wall. With the increase of Richardson number, the position of the maximum velocity in the return flow region gradually deviates from the left wall(Ri $=0.01$, Fig. 4 (b) and Ri =0.02, Fig. 4 (c) ). Meanwhile cause a high streamline curvature within the mixing region.

Figure 5 shows the comparison of the decay of downward velocity predicted by five RANS models at a distance of $14 \mathrm{~mm}$ from the jet wall with the experimental results of $\mathrm{He}$ et al. (2002) for different values of Richardson number. Where the $\mathrm{X}$-axis is the distance of the jet streamwise direction at a distance of $14 \mathrm{~mm}$ from the jet wall, and the $\mathrm{Y}$-axis is the Y-direction component of the jet average velocity. According to the distance of jet stream direction, three stages can be identified. Below the jet exit there is a development stage in which the velocity decays relatively slowly. Further down, at a distance which depends on Richardson number, the decay of velocity becomes much greater due to mixing. Eventually the velocity becomes negative, indicating that the region of the counter-current flow has been reached (He et al. 2002). With the increase of Richardson number, the distance of streamwise direction from the first stage transition to the second get shorter.

It is seen from Fig.4(a) that most of the RANS models tested can capture the shape of the dimensionless vertical velocity profile quite well and the maximum return flow velocity occurs near the left wall in isothermal case. As shown in the Fig.5(a), the distance of development region predicted by most of the RANS models is longer than experimental results, which lead to jet decay occurring slower and a deeper penetration of the jet. The experimental distance of mixing stage ranged between 0.4 to 0.6 as shown in the Fig.5(a). Among

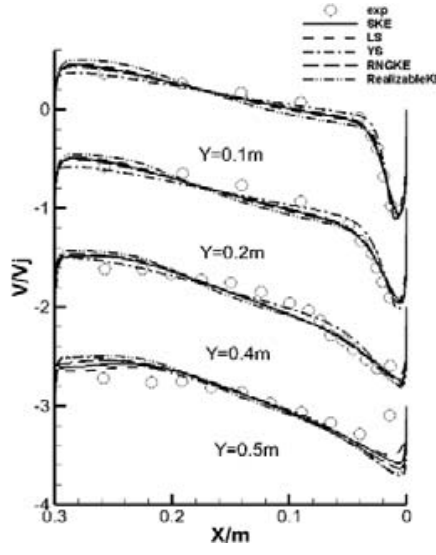

a) $\mathbf{R i}=\mathbf{0}$

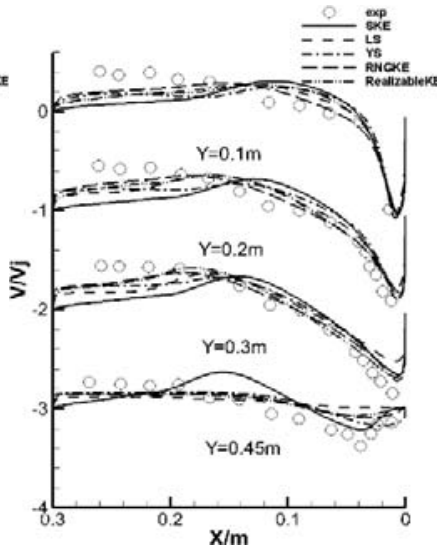

b) $\mathrm{Ri}=0.01$

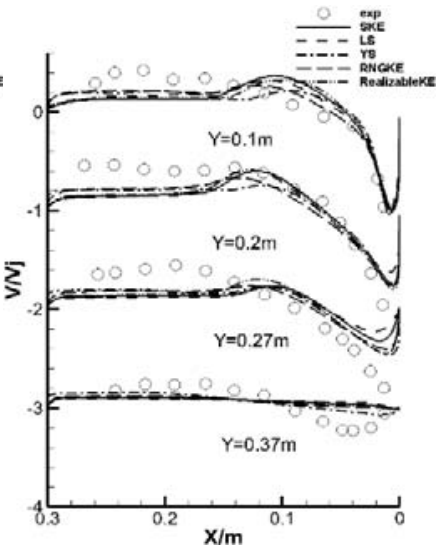

c) $\mathrm{Ri}=\mathbf{0 . 0 2}$

Fig. 4. Comparison of the variation of vertical mean velocity predicted by five RANS models with the experimental results. 


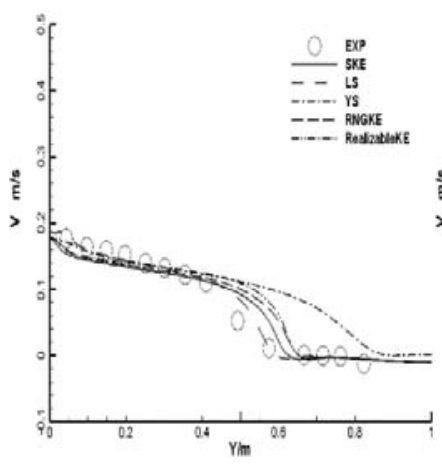

a) $\mathbf{R i}=\mathbf{0}$

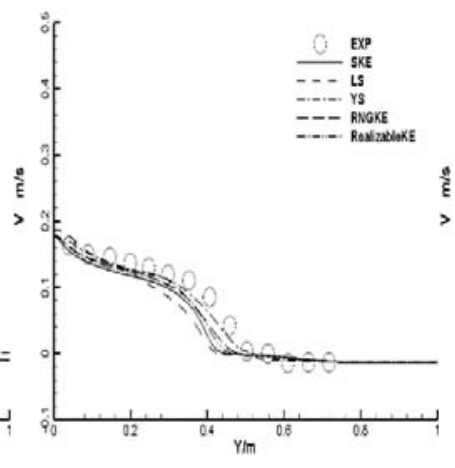

b) $\mathrm{Ri}=\mathbf{0 . 0 1}$

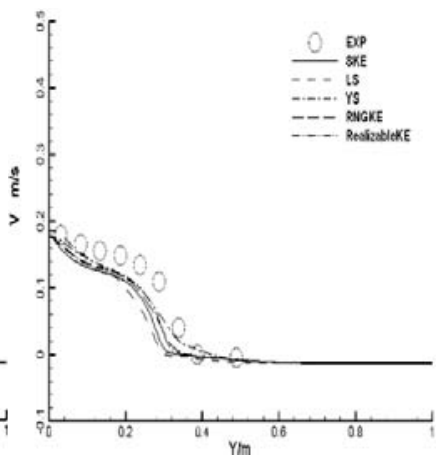

c) $\mathbf{R i}=\mathbf{0 . 0 2}$

Fig. 5. Profiles of decay of downward velocity at a distance of $14 \mathrm{~mm}$ from the jet wall for different values of Richardson number.

those models, the location of mixing stage predicted by LS model show a good agreement with experimental results, which further lead to a satisfactory results for jet velocity profile. See Fig. 4(a) and Fig. 5(a) for details. The good performance of LS may owe to the turbulent viscous modified by the additional source terms $\mathrm{D}$ and $\mathrm{E}$ in transport equation of turbulent kinetic energy and dissipation rate (Launder et al. 1974, Craft et al. 2004). Compared with other RANS models, the location of mixing stage predicted by YS model is relatively backward and this result is similar to the literature.

Due to the modified damping function $f_{1}$ in the YS model is smaller than other RANS models(see Table2 for detail) (Yang and Shih 1993), turbulent viscous calculated by YS model is relatively small (Zhang et al. 2008). See Fig. 3(a) and Fig. 5(a) for details.

As shown in the Fig. 5(b)(c), with the increase of Richardson number (Ri), the distance of streamwise direction from the first stage transition to the second get shorter. The experimental location of mixing stage ranged between 0.36 to 0.48 for $\mathrm{Ri}=0.01$ and 0.25 to 0.4 for $\mathrm{Ri}=0.02$ respectively. However, the location of mixing stage predicted by most of RANS models is more forward in the buoyancy case which lead to jet decay early. Meanwhile, with the increase of Richardson number, the deviation between experiment and numerical simulation get more obvious. Due to the effect of buoyancy, the position of maximum return flow velocity is gradually deviate from the left wall as shown in the Fig. 4(b)(c). However, all RANS models underestimated the effect of buoyancy on the lateral diffusion of jet, and further cause the maximum return flow velocity occurs near the right wall. On the one hand, the instantaneous fluctuation information of flow filed and temperature filed in mixing region is eliminated by time-averaged method. On the other hand, due to the assumption of turbulent normal stress isotropy of $k-\varepsilon$ models (Nie et al. 2017), the effect of buoyancy is dissipated prematurely.

Meanwhile, it is noted that standard $k-\varepsilon$ model overestimated the effect of the buoyancy on the flow filed of mixing region under $\mathrm{Ri}=0.01$ condition (see Fig. 4(b), $Y=0.45$ for detail) and has a relatively big deviation with experimental results. Instead, the RNG $k-\varepsilon$, Realizable $k-\varepsilon$ model and Low-Reynolds Number $k-\varepsilon$ models don't exit this problem. It's interesting to note that the performance of LS and YS model in buoyancy case is complete opposite of isothermal case. Compared to LS model, the position of development stage transition to mixing stage predicted by YS model shows a better consistence with experimental results. See Fig. 5(b), (c) for detail.

Figure 6 shows the comparison of dimensionless wall temperature change predicted by five RANS models with the experimental results of $\mathrm{He}$ et al. (2002) for different values of Richardson number. Where the $\mathrm{X}$-axis is the distance of the jet wall, and the $\mathrm{Y}$-axis is the dimensionless temperature. The $T_{j}$ is jet temperature, and the $T_{c}$ is background flow temperature. These are generally similar to the distributions of velocity in the jet flow shown earlier in Fig. 5. As shown in the Fig. 6, the wall temperature decreases slowly in the development stage and sharply in the mixing stage until it is consistent with the temperature of counter- current stream. All RANS models can predict roughly tendency of wall temperature change. However, it's noted that there exit a huge difference in the transition position of different stage between calculation results and experimental results. Compared to smooth transition in experimental results, there are two obvious turning point in calculation results. In the mixing stage, a very concentrated mixing layer was formed at the interface between the jet and counter-current stream. The turbulence field was strongly modified in this region, the intensity of turbulence peaked and turbulent shear stress changed sign (He et al. 2002). 




a) $\mathbf{R i}=\mathbf{0 . 0 1}$



b) $\mathrm{Ri}=\mathbf{0 . 0 2}$

Fig. 6. Decay of non-dimensional wall temperature along the wall.

Meanwhile, the flow field and temperature field varied with time in a highly intermittent manner. However, as previously mentioned, due to the assumption of isotropy, the dimensionless wall temperature predicted by $k-\varepsilon$ model decreases linearly with a large gradient and shows a obvious deviation from the experimental data. On the other hand, due to the assumption of the modeling of turbulent heat flux using simple gradient diffusion hypothesis by turbulent Prandtl number, the accuracy of temperature field largely depends on the calculation results of flow field. But in complex flow phenomena, a simple turbulent Prandtl number can not accurately reflect the transport state of heat flux (Sommer et al. 1992, Kays et al.1994).

\subsection{LES models}

Figure 7 shows the comparison between the calculated results by LES models and the experimental data of the vertical dimensionless velocity components at at various heights in the test section. Where the $\mathrm{X}$-axis is the distance of the transverse flow field, and the $\mathrm{Y}$-axis is the $\mathrm{Y}$ direction component of the jet average velocity. Figure 8 and Fig. 9 shows comparison of profiles of the horizontal and vertical components of RMS velocity fluctuation with the experimental results of He et al. (2002) respectively. Where the $\mathrm{X}$-axis is the distance of the transverse flow field, and the Y-axis is the vertical and horizontal components of RMS velocity respectively. Figure 10 shows the comparison of the decay of downward velocity. predicted by LES models at a distance of $14 \mathrm{~mm}$ from the jet wall with the experimental results of $\mathrm{He}$ et al. (2002) for different values of

Richardson number. Where the $\mathrm{X}$-axis is the distance of the jet streamwise direction at a distance of $14 \mathrm{~mm}$ from the jet wall, and the Y-axis is the Y-direction component of the jet average velocity.

It is seen from Fig. 8 that the vertical components of RMS velocity fluctuation exits two obvious peaks in

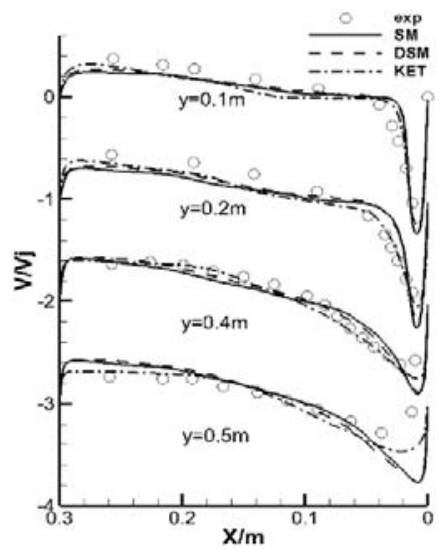

a) $\mathbf{R i}=\mathbf{0}$

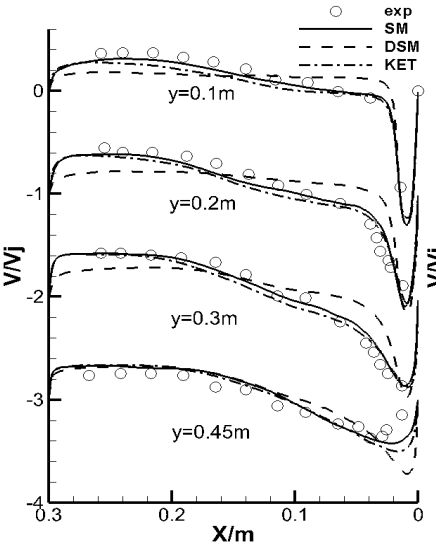

b) $\mathbf{R i}=\mathbf{0 . 0 1}$

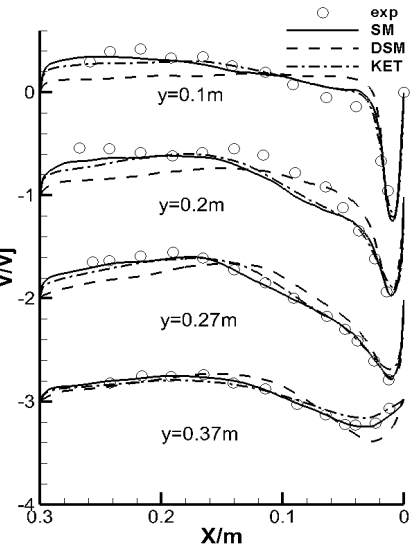

c) $\mathbf{R i}=\mathbf{0 . 0 2}$

Fig. 7. Comparison of the variation of vertical mean velocity predicted by three LES models with the experimental results. 
the wall jet region near the right wall. One peak is located in the outer shear layer far away from the wall and the velocity reaches a maximum here, and the other located in the inner shear layer near the wall is caused by the burst of turbulent vortices near the viscous bottom. The three LES models all can predict this phenomenon, and the calculation results predicted by KET models have an excellent agreement with experimental results. See Fig.8, Fig. 9 for detail.

Compared to the experimental results, the location of mixing stage predicted by SM and DSM models is more backward in the isothermal case as shown in Fig. 10(a). Meanwhile, SM and DSM models underestimate the decay of jet in mixing region, which further lead to the depth of penetration of the downward jet bigger than experimental results as shown in Fig. $7(\mathrm{a}), \mathrm{Y}=0.4 \mathrm{~m}$ and $\mathrm{Y}=0.5$. Instead, although KET model still overestimated the penetration of the downward jet, the location of mixing stage predicted by KET model almost coincides with the experimental results and further KET model can simulate the detail of entire flow field well, as shown in Fig. 7(a), 8(a), 9(a).

With the increase of Richardson number, the distance of streamwise direction from the first stage transition to the second get shorter. It's noted that the location of mixing stage and vertical dimensionless velocity components predicted by SM and KET model are very close to the experimental results as shown in Fig. 7(b), (c). The entire vertical component flow filed predicted by DSM exit an obvious deviation with experimental results near the left wall. With the increase of buoyancy effect, the velocity of entire flow filed decreases which further lead to the decay of turbulence fluctuation. Among this three LES models, KET model can grasp this change and provide accurate turbulence information, see Fig. 7(b)(c), Fig. 8(b)(c), Fig. 9(b)(c) for detail.

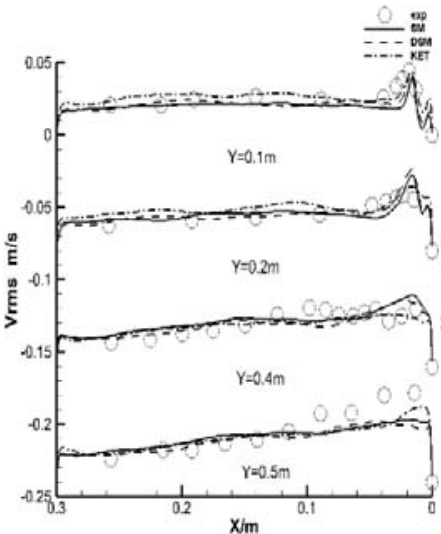

a) $\mathbf{R i}=\mathbf{0}$

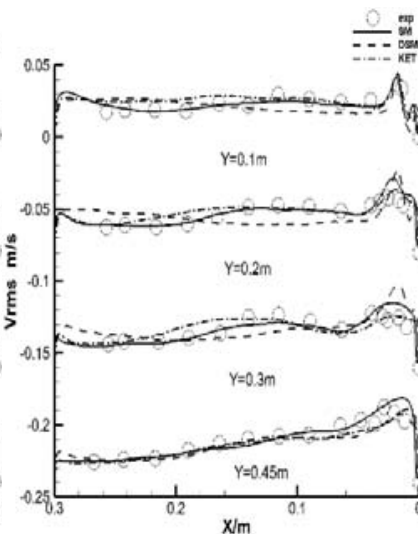

b) $\mathrm{Ri}=\mathbf{0 . 0 1}$

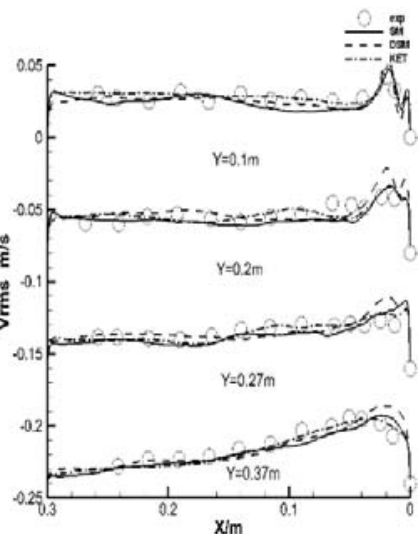

c) $\mathrm{Ri}=0.02$

Fig. 8. Comparison of profiles of vertical components of RMS velocity fluctuation with experimental results.

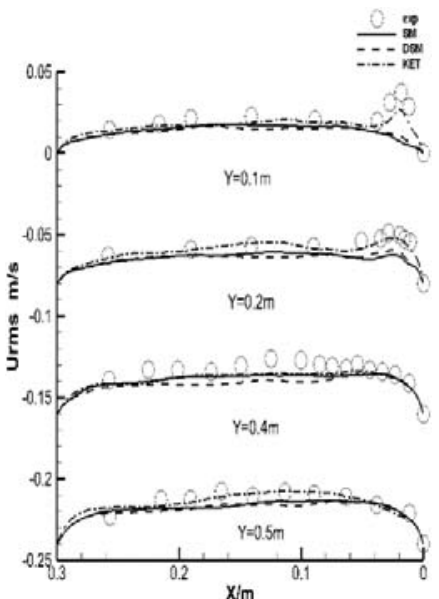

a) $\mathbf{R} \mathbf{i}=\mathbf{0}$

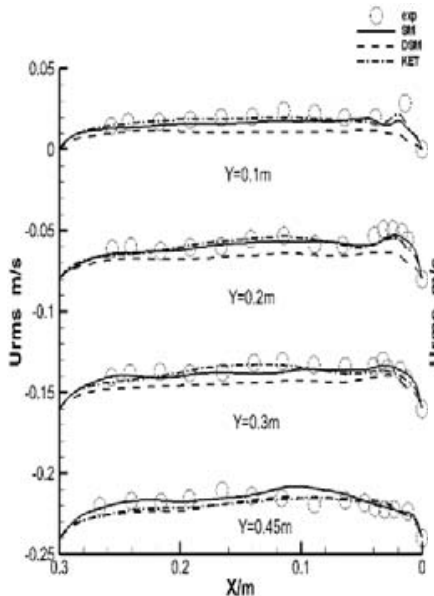

b) $\mathbf{R i}=\mathbf{0 . 0 1}$

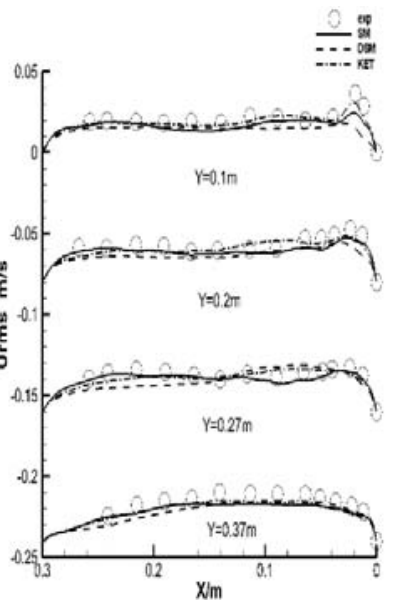

c) $\mathrm{Ri}=\mathbf{0 . 0 2}$

Fig. 9. Comparison of profiles of the horizontal components of RMS velocity fluctuation with the experimental results. 


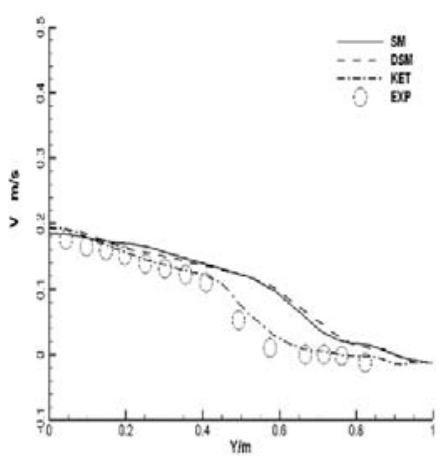

a) $\mathbf{R i}=\mathbf{0}$

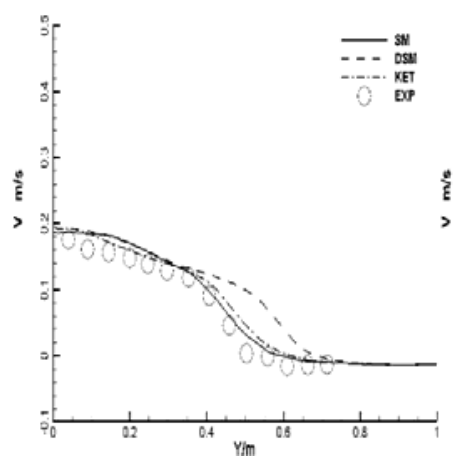

b) $\mathbf{R i}=\mathbf{0 . 0 1}$

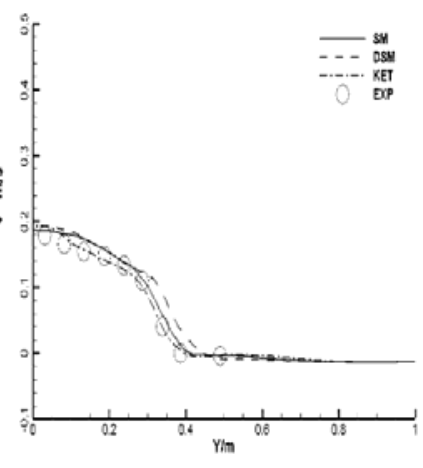

c) $\mathbf{R i}=\mathbf{0 . 0 2}$

Fig. 10. Profiles of decay of downward velocity at a distance of $14 \mathrm{~mm}$ from the jet wall for different values of Richardson number.

Compared to RANS models, LES models show the better capability for simulation of buoyancy opposed wall jet. In mixing region, LES models consider the buoyancy effect on instantaneous turbulence fluctuation information so that give a satisfactory flow filed. In particular, the horizontal and vertical components of RMS velocity fluctuation provided by KET model have an excellent agreement with experimental results.

Due to the turbulent stress term solving by different subgrid-scale models, the turbulent viscosity calculated by the LES models also exit difference. The tendency of jet decay in wall jet region shows the turbulent viscosity coefficient calculated by SM and DSM models is slightly small which lead to a deeper penetration of the downward jet. The Smagorinsky constant $C_{\mathrm{s}}$ in SM model is key point to simulate a flow phenomenon successfully. For this issue, different literatures have recommended different value (C., J. and Doolan 2014, Uddin and Mallik 2015). In this paper, the Smagorinsky constant $C_{\mathrm{s}}$ in SM model was taken value as 0.1 in all simulation condition, which may lead to a relative small flow dissipation and a slightly small turbulent viscosity coefficient in isothermal case. On the contrary, due to effect of buoyancy, $C_{\mathrm{s}}=0.1$ may get closer to realistic flow phenomenon in buoyant case. Hence, the selection of the Smagorinsky constant is not merely depended on empirical judgment according to flow phenomenon, but also needs to make some adjustments base on calculation results. Obviously, it's a limitation of SM model.

DSM model is the dynamic version of SM model using the algorithm proposed by Germano with least squares. It automatically adjusts the Smagorinsky constant at each point in space and at each time step, and avoids the use of Van Driest damping functions. Meanwhile, this method requires a uniform flow field in streamwise(Kim et al. 1997, Liu et al. 2018). However, there exit the interaction of buoyancy with the momentum of the jet in the mixing region where the wall jet flow encounter the counter-current stream, which leads to the strong turbulence shear stress and non uniform flow field. With the increase of buoyancy, this flow characteristic will be more obvious. It also explains the reason that performance of DSM model is worse than SM model in buoyant case.

Compared to SM and DSM models, KET model doesn't exit the issue about the Smagorinsky constant. When KET model calculate the instantaneous flow field in the mixing region, the subgrid-scale model and filtering scale can be dynamically adapted by adjusting $C_{\mathrm{k}}$ and $C_{\varepsilon}$ in the subgrid turbulent energy transport equation (Kim et al. 1997). Due to the subgrid turbulent energy transport equation, the turbulent shear stress and viscosity coefficient calculated by KET model is more approach to the realistic transport characteristic of turbulent energy and reflects the non-equilibrium of turbulent flow field.

Figure 11 shows the three dimensional pressure isosurface contour calculated by LES models in buoyant case for $\mathrm{Ri}=0.02$. It can be seen obviously in the Fig.11 that a very concentrated mixing layer was formed at the interface between the jet and counter-current stream and there exit many turbulent eddy structure with different scales.

Figure 12 shows the comparison of dimensionless wall temperature change predicted by LES models with the experimental results of $\mathrm{He}$ et al. (2002) for different values of Richardson number. Where the Xaxis is the distance of the jet wall, and the $\mathrm{Y}$-axis is the dimensionless temperature. The $T_{j}$ is jet temperature, and the $T_{c}$ is background flow temperature. Compared to RANS models, the entire tendency of wall temperature change predicted by LES models have an excellent agreement with experimental results. Due to the advantage of the direct solution for large scale vortices, LES models can simulate a accurate flow field and temperature field especially in mixing region where the buoyancy effect is significant and the temperature field varied with time in a highly intermittent manner. It's noted that the dimensionless wall temperature predicted by LES models is higher than experimental results and 




$\mathrm{Ri}=0.02: \quad$ a) $\mathrm{SM}$ model



b) DSM model

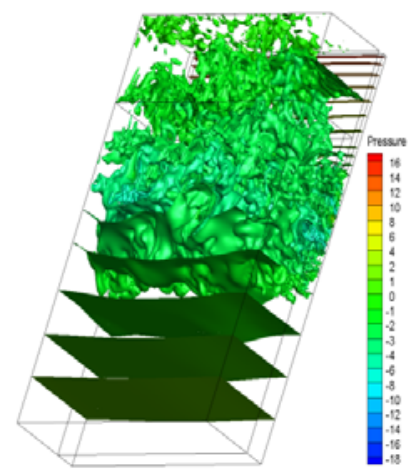

c) KET model

Fig. 11. Three dimensional pressure isosurface contour.

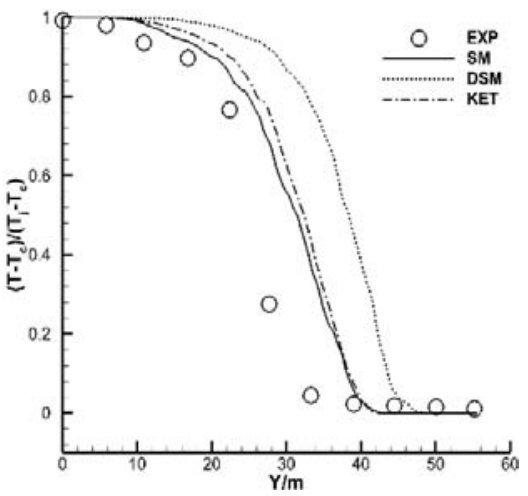

a) $\mathbf{R i}=\mathbf{0 . 0 1}$



b) $\mathrm{Ri}=\mathbf{0 . 0 2}$

Fig. 12. Decay of non-dimensional wall temperature along the wall.

this deviation is gradually reducing with the increase of Richardson number. This may be due to the difference between surrounding environment and counter-current stream, the additional heat loss in the experiment is smaller with the increase of Richardson number. On the other hand, the vertical walls in numerical simulation are set as adiabatic. Those factors result in a little deviation between simulation and experiment together. Among this three LES models, SM model and KET model can better capture the variation trend of wall temperature, while DSM model overestimates the wall temperature, as shown in Fig. 12a) and b).

\section{CONCLUSION}

A comparative study of buoyancy-opposed wall jet carried out using RANS models and LES models. The calculation results were compared with the available experimental data. Some of the specific conclusions are summarized as follows:

- In isothermal case, the RANS models tested in this paper can predict accurately the flow field information and have engineering application value.
Due to the additional source term $\mathrm{D}$ and $\mathrm{E}$ in turbulent transport equation, LS model can capture the position of mixing stage. Instead, the location of mixing stage predicted by YS model is relatively backward because of the modified damping function $f_{1}$.

- In buoyant case, there exit a turbulence field strongly fluctuated and flow field with anisotropy in mixing region. The $k-\varepsilon$ models can not simulate flow field temperature field in this region well due to the Reynolds time average method and the assumption of turbulent normal stress isotropy. For LES models, due to the the advantage of the direct solution for large scale vortices, the influence of the anisotropy of the flow field in the mixing region on the temperature field was considered. The LES models are more suitable for this flow phenomenon.

- Regarding the relative performance of various LES models tested, due to the turbulent shear stress and viscosity coefficient calculated by subgrid turbulent energy transport equation is more approach to the realistic transport characteristic of turbulent energy, the KET model is capable of predicting the 
influence of buoyancy effect on flow field and temperature field in good agreement with experimental results over the all flow conditions.

\section{REFERENCES:}

Addad, Y., S. Benhamadouche and D. Laurence (2004). The Negatively Buoyant Wall-Jet: LES Results. International Journal of Heat \& Fluid Flow 25, 795-808.

Craft, T. J., A. V. Gerasimov, H. Iacovides and B. E. Launder (2002). Progress in the Generalization of Wall-Function Treatments. International Journal of Heat And Fluid Flow 23, 148-160.

Craft, T. J., A. V. Gerasimov, H. Iacovides, J. W. Kidger and B. E. Launder (2004). The Negatively Buoyant Turbulent Wall Jet: Performance of Alternative Options in RANS Modelling. Heat Fluid Flow 25, 809-823.

Doolan, C. J. (2014). Large Eddy Simulation of the Near Wake of a Circular Cylinder at SubCritical Reynolds Number. Engineering Applications of Computational Fluid Mechanics.

Germano, M., U. Piomelli, P. Moin and W. H. Cabot (1991). A Dynamic Sub-Grid-Scale Eddy Viscosity Model. Journal of Fluid Mechanics $86,491-511$.

Goldman, D. and Y. Jaluria (1986). Effect of Opposing Buoyancy On the Flow in Free and Wall Jets. Journal of Fluid Mechanics 166, 4156.

Guo, Y., C. X. Xu, G. X. C. Z. S. Zhang (2006). Large Eddy Simulation of Scalar Turbulence Using a New Subgrid Eddy Diffusivity Model. International Journal of Heat \& Fluid Flow 28, 268-274.

He, S., Z. Xu and J. D. Jackson (2002). An Experimental Investigation of BuoyancyOpposed Wall Jet Flow. International Journal of Heat \& Fluid Flow 23, 487-496.

Huai, W. X (2010). Numerical Simulation of Horizontal Buoyant Wall Jet. Journal of Hydrodynamics 22, 58-65.

Jones, D. A. and M. Chapuis (2016). RANS Simulations Using OpenFOAM Software. Defence Science and Technology Group, 139151.

Kapoor, K. and Y. Jaluria (1989). Heat Transfer From a Negatively Buoyant Wall Jet. International Journal of Heat \& Mass Transfer 32, 697-709.

Kapoor, K. and Y. Jaluria (1991). Mixed Convection Flow Due to a Buoyant Wall Jet Turning Downward at a Corner. Mixed Convection Heat Transfer 163, 119-128.

Kays and M. William (1994). Turbulent Prandtl
Number-Where are we?" Asme Transactions Journal of Heat Transfer 116, 284-295.

Kim, W. W., S. Menon, W. W. Kim and S. Menon (1997). Application of the Localized Dynamic Subgrid-Scale Model to Turbulent WallBounded Flows. American Institute of Aeronautics and Astronautics, 1-13.

Launder, B. E. and B. I. Sharma (1974). Application of the Energy-Dissipation Model of Turbulence to the Calculation of Flow Near a Spinning Disc Pergamon 2, 131-138.

Li, Z. W, W. X. Huai (2011). Large Eddy Simulation of the Interaction Between Wall Jet and Offset Jet. Journal of Hydrodynamics 23, 544-553.

Li, Z. W, W. X. Huai, Z. D. Qian (2021). Study On the Flow Field and Concentration Characteristics of the Multiple Tandem Jets in Crossflow. Science China Technological Sciences 55, 2778-2788.

Liu, Q. L., C. He and H. X. Lai (2018). Large Eddy Simulation of a Plane jet and Comparison of SGS Models. Journal of Engineering Thermophysics 6, 1272-1278.

Meng, G., H. Wenxin, X. Yizhou, Y. Zhonghua and J. Bin (2018). Large Eddy Simulation of a Vertical Buoyant Jet in a Vegetated Channel. International Journal of Heat \& Fluid Flow 70, 114-124.

Nie, X. and Y. Z. Zhang (2017). Comparative Analysis and Numerical Simulation About Six Low Reynolds Number k- $\varepsilon$ Models in Nearwall Shear Flow. Proceedings of the CSEE 24, 7247-7254.

Nie, X., Z. H. Zhu, H. B. Liao, M. Lv and X. R. Xu (2021). A Comparative Study of Heat Transfer Characteristics of Wall Jet with Boundary Layer Transition Using Six low-Reynolds Number K-E Models. AIP Advances 11, 25025.

Rathore, S. K. and M. K. Das (2016). Numerical Investigation On the Performance of lowReynolds Number K- Model for a BuoyancyOpposed Wall Jet Flow. International Journal of Heat \& Mass Transfer 95, 636-649.

Shih, T. H., W. W. Liou, A. Shabbir, Z. Yang and J. Zhu (1995). A New $\kappa-\varepsilon$ Eddy Viscosity Model for High Reynolds Number Turbulent Flows. Computers Fluids 24, 227-238.

Smagorinsky, J. (1963). General Circulation Experiments with the Primitive Equations. Monthly Weather Review 91, 99-164.

Sommer, P. T., M. C. R. So and G. Y. Lai (1992). A Near-Wall Two-Equation Model for Turbulent Heat Fluxes. International Journal of Heat And Mass Transfer 12, 3375-3387.

Speziale, C. G. and S. Thangam (1992). Analysis of 
X. Nie et al. / JAFM, Vol. 15, No. 1, pp. 85-98, 2022.

an RNG Based Turbulence Model for Separated Flows. International Journal of Engineering Science 30, 1379.

Uddin, M. and M. Mallik, (2015). Large Eddy Simulation of Turbulent Channel Flow Using Smagorinsky Model and Effects of Smagorinsky Constants. British Journal of Mathematics \& Computer Science 7, 375-390.

Zhang L J., L. Zhou and X. C. Chen (2008). Numerical Simulation of Flow Around Square
Cylinder Using Different low-Reynolds Number Turbulence Models. Journal of Central South University of Technology 04, 140-144.

Yang, Z. and T. H. Shih (1993). A New Time Scale Based K-Epsilon Model for Near Wall Turbulence. AIAA JOURNAL 31, 1191-1198. 